\title{
A Comparison of Network Level Pavement Condition Assessment in Road Asset Management
}

\author{
Junzhe Wang ${ }^{1}$, , Ming Chen ${ }^{1}$, Wei Gao ${ }^{2}$, Zhenhua Guo ${ }^{2}$, Yangjie Liu ${ }^{2}$ \\ ${ }^{1}$ Engineering Technology and Materials Research Center, China Academy of Transportation Sciences, Beijing, China \\ ${ }^{2}$ Beijing Shoufa Highway Maintenance Engineering Co., Ltd, Beijing, China \\ Email address: \\ junzhe_wang@outlook.com (Junzhe Wang), chenming@motcats.ac.cn (Ming Chen), 406590106@qq.com (Wei Gao), \\ 1042545848@qq.com (Zhenhua Guo),2152621@qq.com (Yangjie Liu) \\ ${ }^{*}$ Corresponding author
}

\section{To cite this article:}

Junzhe Wang, Ming Chen, Wei Gao, Zhenhua Guo, Yangjie Liu. A Comparison of Network Level Pavement Condition Assessment in Road Asset Management. International Journal of Transportation Engineering and Technology. Vol. 6, No. 3, 2020, pp. 95-101. doi: $10.11648 /$ j.jitet.20200603.14

Received: September 2, 2020; Accepted: September 19, 2020; Published: September 23, 2020

\begin{abstract}
Transportation agencies face the challenging task to maintain, preserve and improve infrastructure condition while with limited funding. Pavements are one of the major assets of roadway systems and pavement management system (PMS) are broadly accepted and implemented by agencies and organizations to maintain pavement structures at a high level of service. PMS is a set of tools to support the decision-making process for determining the demand of maintenance, prioritizing projects and optimizing funding allocation. Pavement condition monitoring may be evaluated or assessed by means of various indicators. Performance indicators are an essential part in a PMS, individual performance indicators (IPIs) and combined performance indicators (CPIs) are proposed to monitor and report pavement conditions. IPIs characterize the general condition of the various types of pavement distress which can be related to road performance. The CPI for each road type can be developed or calculated from IPIs. Focus on network level analysis of road pavements, the objective of this paper is to review and compare the development and application of performance indicators for assessment of pavement condition of different country's guidelines. The utilization and integration mechanism of individual indicator are described and compared among selected country guidelines. The prospective indicators and techniques for future application are further discussed. It can be conclude from this study that the majority studied guidelines have placed great emphasis on surface distress and roughness for pavement condition assessment; international roughness index (IRI) is the most commonly used parameter for evaluation of road roughness due to its objectivity while the determination of surface distress is more subjective. The integration methods from IPIs into CPIs can be summarized as "deduct system method", "sum system method", "weighted sum method" and "equation method".
\end{abstract}

Keywords: Roads \& Highways, Maintenance \& Inspection, Pavement Condition, Management

\section{Introduction}

Road networks are one of the most important infrastructure assets that are owned and maintained by local government. The general condition of road network is of great importance of the society since the community heavily rely on the roads. The asset management process provides a systematic and consistent method for selection of maintenance and repair needs by evaluating the pavement performance at the network level. Pavement management systems are broadly accepted and implemented by agencies and organizations with responsibilities for designing, constructing, and maintaining pavement structures [1]. Pavement performance evaluation which identify pavement distress types, severities and quantities thereby providing a condition index or rating are an essential function of any pavement management system.

In order to fulfil the evaluation of pavement condition, performance indicators are required and defined in a pavement management system. Generally, there are individual performance indicators (IPIs) and combined performance indicators (CPIs) proposed to monitor and report pavement conditions [2]. Each IPI represents one characteristic of the pavement which provides useful 
information either structural (deflection) or functional (cracking) to assessor. The IPIs can be further used to form a single index for the characterization of various contribution to the performance of pavement asset. The individual performance indicator is normally gained by direct measurement using appropriate equipment while the combined performance indicator is based on a sum of weightings of the relative importance of each individual performance indicator [3]. The majority performance indicators can be categorized by either evaluation object or function as illustrated in Figure 1. The fundamental reason for having performance indicators is that the limited availability of resources for road infrastructure requires the allocation of these resources as efficient as possible among competing alternatives [4]. More specifically, the majority use of performance indicators focuses on the following aspects: (a) evaluation of road network condition [5]; (b) determination of asset value [6]; (c) effectiveness evaluation of policy [7]; (d) trigger treatments and diagnostic use for appropriate remedial actions [8-9].

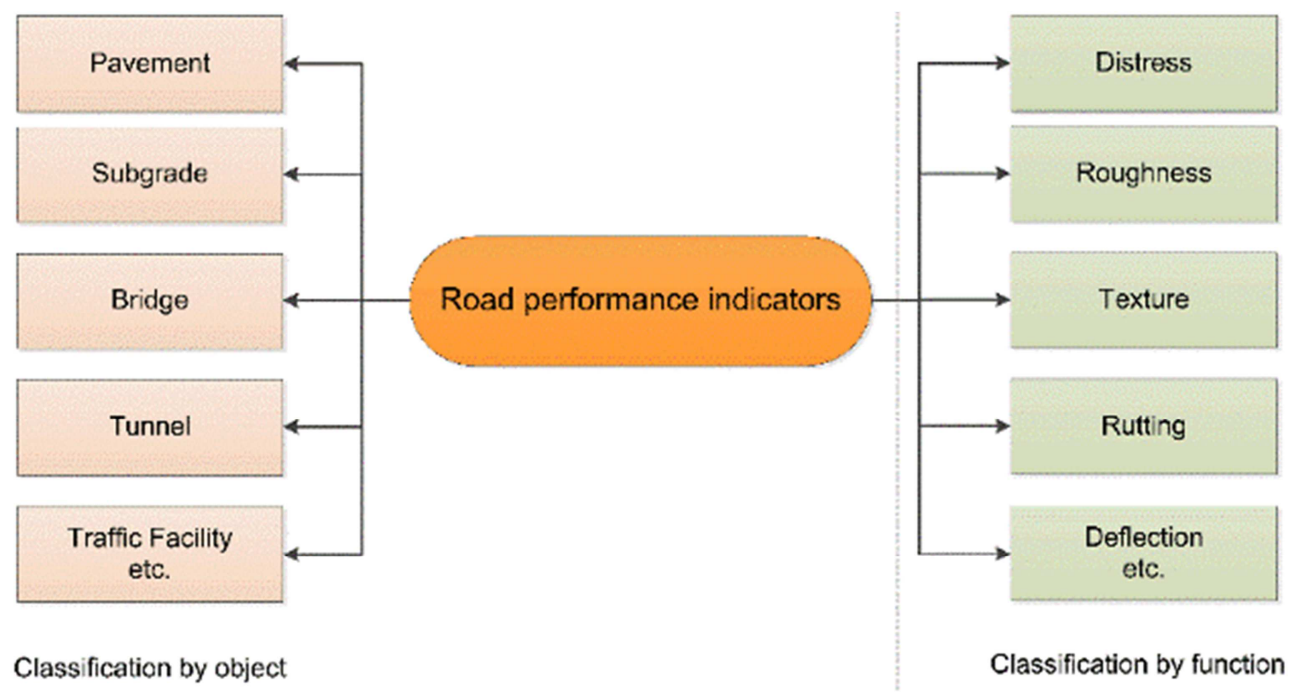

Figure 1. Classification of road performance indicators.

Majority roadway agencies have established their guidelines for evaluation of pavement conditions and various performance indicators were defined and used. The objective of this paper is to review, analyze and compare the road condition assessment guidelines used in different countries and mainly focuses on the evaluation of pavement asset. Generally, the guideline or standard for pavement assessment were formulated by the department of transport in different levels government and applied in a certain administrative area [10-13]. At the same time, there is also a series of standard proposed by professional associations or research institutions which is widely accepted and used by engineers in industry [14-16]. In this paper the characteristics and rules of pavement condition assessment guidelines in New York, Mainland China, Ontario and Scotland as a representative of the US, China, Canada and UK are studied and discussed. It mainly focuses on the utilization and integration of individual indicators in these guidelines. The resulting guidelines for the assessment and maintenance of highways can be used by stakeholders as a reference to international best practice.

\section{Pavement Condition Assessment}

\subsection{China Guidelines}

China initiated its work in pavement performance assessment in 1980s, the Ministry of Transport PRC [17] promulgated interim measures for inspection and evaluation of maintenance quality for national road. In this trial regulation, a deduct value based condition assessment method was proposed. A perfect pavement is assigned with a score of 100, and pavement condition at any point of time is assessed by deducting the cumulative value based on the level of severity and extent of distresses present. On the basis of previous experience, the Ministry of Transport PRC published the first industry standard for inspection and evaluation of highway maintenance quality in 1994 [18]. In this standard, the deduct value based method was inherited and more specific definitions of different distresses were provided. With the development of technologies for data collection, a series of multi-functional equipment have been developed and used to achieve automated highway condition survey. The Ministry of Transport PRC reviewed and made vital revise of the standard and promulgated a revised version of standard in 2007. The new standard focuses the following four aspects in condition assessment: (a) pavement surface distress; (b) riding quality; (c) asphalt rutting; (d) skid resistance ability, and four individual indicators were accordingly established [11]. A combined indicator named pavement quality index (PQI) was developed for comprehensive indication of pavement condition. PQI is a function of four sub-indexes as formulated in Equation 1.

$$
P Q I=\omega_{p c i} * P C I+\omega_{r q i} * R Q I+\omega_{r d i} * R D I+\omega_{s r i} * S R I
$$

Pavement surface condition index (PCI) is designed to 
quantify the pavement distresses including alligator cracking, longitudinal cracking, transverse cracking, pot holes, patching etc. for asphalt pavement and faulting, edge spalling etc. for concrete pavement. PCI is correlated and calculated from a distress ratio (DR), while the distress ratio is defined as the total deterioration area divided by total area of the subsection measured. Riding quality index (RQI) was proposed with intention to rate the driving comfort, it is calculated as a function of international roughness index (IRI). Rutting defect of asphalt pavement was assessed by using rutting depth index (RDI), a sectioned function between rutting depth (RD). Skidding resistance index (SRI) is an indicator reflects the skid resistance ability by measuring side-way force coefficient (SFC). The national standard stipulated these performance indicators and its corresponding weighting factors in terms of different surface type and technical specification as listed in Table 1. It is noted that the rutting depth is only applicable for asphalt pavement of expressway and first-class highway. For other trunk highway, RQI and PCI are the predominate indexes and the calculation of PQI is identical for both asphalt and concrete pavement. Comparing with expressway (first-class highway), the weighting factor of PCI for other trunk road increase from 0.4 to 0.6 while the weighting factor for RQI keeps the same. It is worth mentioning that both combined indicator and individual indicators have a scale ranging from 0 to 100 , with 100 representing a flawless pavement condition.

Table 1. The weighting factor of sub-index for different pavement types.

\begin{tabular}{llll}
\hline & $\begin{array}{l}\text { Weighting } \\
\text { factor }\end{array}$ & $\begin{array}{l}\text { Expressway \& } \\
\text { first-class highway }\end{array}$ & $\begin{array}{l}\text { Other Trunk } \\
\text { highway }\end{array}$ \\
\hline \multirow{3}{*}{ Asphalt } & $\mathrm{w}_{\mathrm{pci}}$ & 0.35 & 0.6 \\
pavement & $\mathrm{W}_{\mathrm{rqi}}$ & 0.4 & 0.4 \\
& $\mathrm{~W}_{\mathrm{rdi}}$ & 0.15 & - \\
& $\mathrm{W}_{\mathrm{sri}}$ & 0.1 & - \\
\multirow{2}{*}{ Concrete } & $\mathrm{w}_{\mathrm{pci}}$ & 0.5 & 0.6 \\
pavement & $\mathrm{W}_{\mathrm{rqi}}$ & 0.4 & 0.4 \\
& $\mathrm{w}_{\mathrm{sri}}$ & 0.1 & - \\
\hline
\end{tabular}

\subsection{Canada Ontario Guidelines}

Ontario's pavement condition assessment method relies on the examination of two principal features: one is the riding quality and the other is distress manifestation. The key performance indicators used by the Ontario Ministry of Transport (MTO) at network level include: (a) IRI, (b) riding comfort index (RCI), (c) distress manifestation index (DMI) and (d) pavement condition index (PCI) [19].

The DMI addresses pavement surface distresses such as cracks, distortion, and other defects. The model for distress manifestation was initially developed under the research work carried out by Phang et al. [20] and Hajek and Phang [21]. It is an equivalent indicator as PCI used in China guideline. The DMI was further revised and adopted by the MTO in its PMS with a definition shown in Equation 2 [22].

$$
D M I=10 * \frac{D M I_{\max }-\sum_{i=1}^{n} \omega_{i}\left(s_{i}+d_{i}\right)}{D M I_{\max }}
$$

Where $\mathrm{i}=$ distress type; $\omega_{\mathrm{i}}=$ weighting factor for a particular type of crack or other form of pavement distress. $\mathrm{s}_{\mathrm{i}}=$ weighting factor for severity of crack or other form of pavement distress; $\mathrm{d}_{\mathrm{i}}=$ weighting factor for density of crack or other form of pavement distress; $\mathrm{DMI}_{\max }=$ the maximum value of an aggregated pavement distresses, defined by pavement type.

The riding quality or roughness of the pavement was initially rated subjectively by using a riding comfort rating (RCR) indicator. The assessment was carried out by a rater in a passenger car travelling at a standard speed of $80 \mathrm{~km} / \mathrm{h}$, and the pavement was rated into five grades namely excellent, good, fair, poor and very poor on a basis from 0 to 10 scale. This method is easy to perform but the results is highly depending on the human judgment which may lead to inconsistencies in the priority list of funds allocation. In order to minimize the human factor and achieve a more objective result, since 1997 MTO has moved to IRI for measurement of roughness and established a correlation between IRI and RCR as shown in Equation 3 [23]. During the upgrade of PMS in 2001, RCR was renamed as RCI and adopted by MTO [19].

$$
R C R=8.52-7.49 * \log _{10} I R I
$$

PCI is MTO's overall measurement of pavement condition, and it is equivalent to PQI when compared with China guideline. It is obtained by combining the DMI and $\mathrm{RCI} / \mathrm{RCR}$ values in a linear model. PCI was initially developed by Hajek and Phang [21] and was used by MTO from 1986 to 2001 with a definition in Equation 4. Where c and $\mathrm{s}$ are constant which is related to the determination method of roughness.

$$
P C I=100 *(0.1 * R C R)^{0.5} \frac{(205-D M I)}{205} * c+s
$$

The determination equation of PCI experienced several revises, with the introduction of IRI, Equation 5 gives the calculation of PCI in MTO PMS up to date:

$$
P C I=a+b * D M I-c * I R I
$$

Where, a, b, and c are coefficients developed through regression analysis for each different pavement types based on historical monitoring data.

\subsection{U.S. New York Guidelines}

One of the earliest pavement condition indices in the US was the present serviceability rating (PSR) developed through the AASHO Road Test in 1950s by having raters riding in an automobile and assigning a pavement condition value [24]. A more objective index called present serviceability index (PSI) was proposed by Carey Jr and Irick consequently [25]. In the US, it is compulsory responsibility of state Department of Transport (DoT) to establish measures to assess the condition and minimum service level of pavements, which is required by law at national level [26]. In 
order to assess network-level pavement condition, a comprehensive indicator also named as PCI which include surface rating, ride quality, and dominant distresses properties was developed by New York state DoT [10].

A deduct system where points for each pavement defect are subtracted from a perfect rating of 100 has been used for the calculation of New York PCI, and this approach shares the same concept as described in ASTM D6433-03 [14]. The maximum deduction points of each evaluation aspect for different pavement types were listed in Table 2.

Table 2. Maximum deduction point for different pavement types.

\begin{tabular}{|c|c|c|}
\hline & Asphalt pavement & Portland cement concrete pavement \\
\hline Surface Rating & 35 & 35 \\
\hline IRI & 35 & 35 \\
\hline Rutting & 15 & 5 \\
\hline Alligator Crack or Widening drop & 15 & - \\
\hline Faulting & - & 15 \\
\hline Spalling & - & 10 \\
\hline
\end{tabular}

The surface distress condition is rated on the severity of cracking on pavement surface and conducted by region-based crews. The rater assigns a rating based on a comparison between what is seen on the pavement and pre-defined photographs of pavements with rating scale. The rating of the photograph most closely representing the average condition of the rating segment is assigned with a scale from 1 to 10 , with 10 indicates an excellent condition of pavement surface with no distress. During the rating survey, the dominant distresses for each road section was identified simultaneously, alligator cracking and widening dropoff are considered for asphalt pavement while faulting and spalling are identified for concrete pavement. Ride quality, rutting and faulting data were collected using high speed profiler survey equipment, and corresponding processing procedures follow the AASHTO practice standard R43, R48 and R36 respectively [27-29]. Based on collected data, the determination of deduct value for IRI, rutting and faulting can be derived from Equations 6 to 8:

$$
\begin{gathered}
I R I: \mathrm{y}=1.08 *\left(9 E^{-6 *} I R I^{3}-0.0075^{*} I R I^{2}+2.0532 * I R I-154.37\right) \\
I R I: \mathrm{y}=0.90 *\left(2 E^{-6 *} I R I^{3}+0.0004^{*} I R I^{2}+0.0596^{*} I R I-5.2585\right) \\
\text { Rutting }: \mathrm{y}=-73.81 * R D^{3}+91.429 * R D^{2}-6.9762^{*} R D-0.1429 \\
\text { Faulting }: \mathrm{y}=83.333^{*} H^{3}-125^{*} H^{2}+71.667 * H+1 E^{-13}
\end{gathered}
$$

Two equations are provided for the IRI deduct because it was difficult to get a single equation to fit the curve well. It is noted that there is also a 5 points for rut depth deduct of Portland cement concrete pavement and it is obtained by dividing the resulting equation deduct value by 3 to achieve a maximum point of 5 . Compared with IRI and rutting, the scale for surface rating including alligator crack and spalling is subjected to the judgment of experts and deteriorated intuitively though the scoring system, therefore are partial to be more subjective.

\subsection{UK Scotland Guidelines}

There are two major programs for road condition survey in the UK, on the trunk road network in England, surveys were carried out as Traffic Speed Condition Survey (TRACS) program, while on the national network in Scotland, designated roads in Northern Ireland and local authority road network in England, the Surface Condition Assessment of the National Network of Roads (SCANNER) system were preferred [13].

A road condition indicator ( $\mathrm{RCI}$ ) was developed to characterize overall condition of carriageway in Scotland under SCANNER system. The proposal of this indicator has experienced a process of research, development, testing and refinement stages. Based on preliminary research on TRACS type survey defects index carried out by Cartwright and Pickett [30], original SCANNER condition indicator parameter including thresholds and weightings have been proposed and used in principal road during the national survey in 2005/2006 in Scotland [31]. After a review of the results with the initial values and further tests on classified roads, a revised set of values were used for later surveys [32].

The evaluation of the pavement condition in Scotland includes features of roughness, rut depth, cracking and texture depth. Each feature has been allocated a maximum point correlated with individual parameter, and the maximum point and weighting factor of revised RCI were listed in Table 3. Each parameter is scored linearly between a lower threshold and an upper threshold, while these thresholds were based on engineers' experience, and has a value from zero at the lower threshold to 100 at the upper threshold. Figure 2 gives an example of scoring rut depth parameter. The threshold values for each parameter can be referenced to the SCANNER surveys guide and specification [33]. 


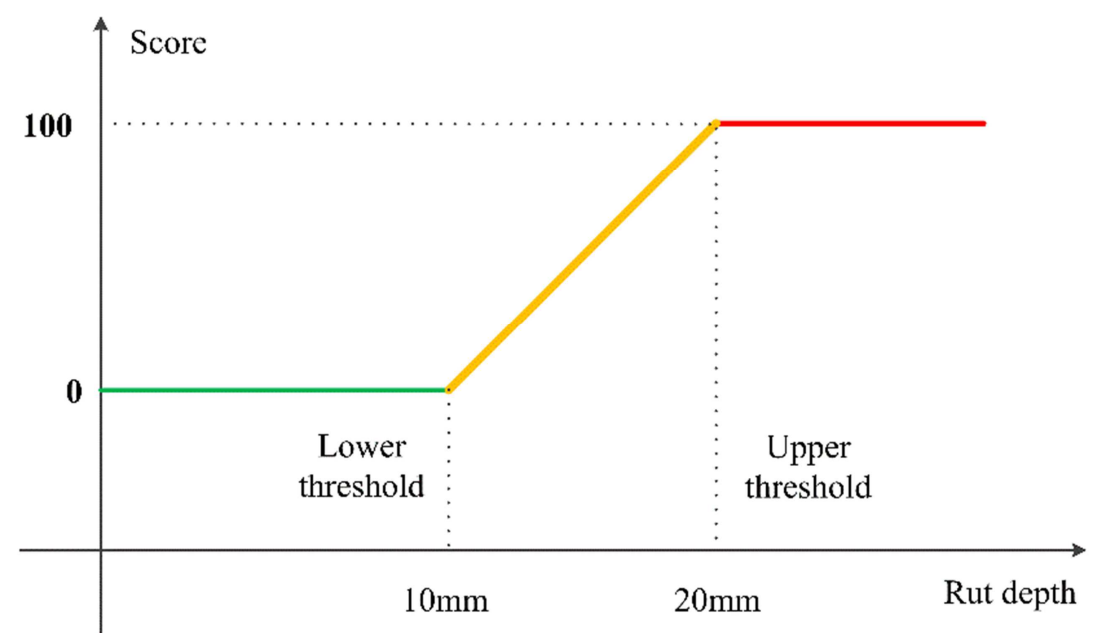

Figure 2. Example of scoring a SCANNER parameter-rut depth.

The scores of each parameter are then summed for each nominal $10 \mathrm{~m}$ subsection of the survey, giving a value between zero (indicates a new/nearly perfect condition) and
315 (indicates sever deterioration condition) for quantifying pavement condition.

Table 3. Maximum point and weighting factors of individual parameter for revised RCI.

\begin{tabular}{llll}
\hline & Parameter & Weighting factor & Maximum point \\
\hline & 3m longitudinal profile variance & 0.8 & \\
Roughness & 10m longitudinal profile variance & 0.6 & 1.0 \\
Rutting & rut depth & 0.6 & 100 \\
Surface distress & whole carriageway cracking intensity & varies 0.3 to 0.75 & 60 \\
Texture & sensor measured texture depth (SMTD) & & 75,50 or 30 \\
Maximum total points & & & 315 to 270 \\
\hline
\end{tabular}

\section{Comparison and Discussion}

\subsection{Utilization of Individual Indicators}

In the studied version of China, US, UK and Canada guidelines, it is learned that a combined indicator has been established in all guidelines, and 4, 6, 6, 2 individual indicators were used respectively in corresponding country/state guidelines. The individual indicators utilized in each guideline can be categorized into five groups and weighting factor for each group is calculated and summarized in Table 4 for comparison. It is noted that the weighting factor for expressway of China guideline is selected for comparison purpose, faulting and spalling were considered as part of surface distress when calculating the weighting factor for New York guideline, and the weighting factor of each group for Scotland was calculated based on the maximum points of each indicator with an assumption of the total points are 315. Regarding the information shown in Table 4, the following observations are worth mentioning: (a) Except for Ontario guideline, each group of the individual indicator has been assigned a constant value which may vary from pavement types, while the weighting factor/parameter in Ontario guideline are determined through regression analysis based on historical data; (b) The surface distress and roughness condition are proved to be essential and important for pavement condition assessment, the proportion of indicators for these two features has a minimum value of 0.44 in
Scotland guideline, and a maximum value of 0.95 for concrete pavement in New York; (c) The evaluation of pavement rutting has been considered in China, New York and Scotland guidelines, however something is not consistence with the common sense which rutting is especially for asphalt pavement, New York guideline has set up a 5\% proportion for concrete pavement; (d) Majority guidelines adopt IRI for the evaluation of pavement riding quality, while the Scotland guideline defined $3 \mathrm{~m}$ and $10 \mathrm{~m}$ longitudinal profile variance parameters to reflect road roughness.

Table 4. Proportion of individual indicator in different countries guidelines.

\begin{tabular}{lllll}
\hline & China & Ontario & New York & Scotland \\
\hline Surface distress & 0.35 & varies & $0.5 / 0.6$ & 0.19 \\
Roughness & 0.40 & varies & 0.35 & 0.25 \\
Rutting & 0.15 & - & $0.15 / 0.05$ & 0.32 \\
Texture & - & - & - & 0.24 \\
Skidding resistance & 0.1 & - & - & - \\
\hline
\end{tabular}

\subsection{Integration of Individual Indicator}

It is learned that the IPIs can be integrated to form a single index for the characterization of pavement asset. Through the review of above guidelines, variations have exhibited towards the integration mechanism, and the following four methods can be summarized as: "deduct system method", "sum system method", "weighted sum method" and "equation method". China guideline uses the weighted sum method by assigning weighting factors with a sum of 1.0 for each individual indicator. The deduct system method where 
points for each pavement defect are subtracted from a perfect rating of 100 has been used by New York guideline. In contrary of the deduct system method, the Scotland guideline sums up points for each individual parameter with the maximum score indicates the most serve condition of pavement. Ontario guideline established a specific equation for calculation of combined indicator with proportional parameters determined by historical data. Above mentioned integration methods are quite representative and cover majority state or organization guidelines.

\subsection{Next Generation Indicators}

The reviewed guidelines present the experience of pavement condition assessment in different countries, while studies on the how to determine the road condition more precisely and comprehensively have always been a research interest. Two types of methodologies are commonly utilized in this area: the first approach is by introducing new indicators to assess the road condition more comprehensively; In a recently revised version of China highway performance assessment standards, new individual indicators named as pavement bumping index (PBI) and pavement surface wearing index (PWI) are introduced to examine the isolated "bumps" which affect ride quality and macro texture depth properties [34]. In a research on network performance assessment sponsored by Austroads, indicators or examination of environment, safety and efficiency conditions have been proposed [2].

The second approach is by introducing new processing techniques to interpret the data more precisely and confidently Research effort have been made by FHWA on the development of measures to improve confidence levels for pavement condition data including cracking, rutting and faulting etc. [35]. A new approach for the development of pavement condition indicators using a machine learning algorithm has been presented by Marcelino et al. [36]. Research work have been performed by Dong et al. [37] sing artificial neural network technology in order to accurately predict IRI by capturing the latent relationship between the cross-sectional and time-series features.

\section{Conclusion}

In this paper, a review of UK, US, China and Canada guidelines for the pavement condition assessment at network level was presented. Emphasis was placed on the evolution of comprehensive performance indicator, and the differences among the guidelines on utilization and integration of individual indicators were examined. Prospect indicators and techniques for overall assessment of pavement condition for future application were further discussed. Based on the above review, the authors would like to emphasize the following points.

(a) Majority guidelines have placed great emphasis on surface distress and roughness for pavement performance assessment, and IRI is the most commonly used parameter for evaluation of road roughness due to its objectivity; (b) "Deduct system method", "sum system method", "weighted sum method" and "equation method "are typical mechanisms for integration of individual indicators into combined indicator, the weighting factors/points for individual indicators can be either assigned by an expert committee with constant value or through regression analysis based on historical data;

(c) The evaluation of pavement rutting is generally considered for asphalt pavement, while it is also applicable to the examination of concrete pavement;

(d) Additional indicators and new techniques are appropriate approaches to improve accuracy on interpretation and prediction pavement condition and support selection and prioritizing projects for efficient use of funding.

\section{References}

[1] R. Haas, W. Hudson and J. Zaniewski. Modern pavement management systems. Krieger Publishing Company, Florida, USA, 1994.

[2] C. Chin, T. Martin, P. Robinson et al. Network performance indicators - next generation. Austroads Ltd., Sydney, Australia, 2011, Report No. AP-T176/11.

[3] R. Robinson, U. Danielson and M. S. Snaith. Road maintenance management: Concepts and systems. Macmillan Press Limited, Basingstoke, UK, 1998.

[4] R. Haas, G. Felio, Z. Lounis et al. Measurable performance indicators for roads: Canadian and international practice. In Annual Conference of Transportation Association of Canada. (Transportation Association of Canada (ed)). Transportation Association of Canada, Vancouver B. C., Canada, 2009.

[5] G. Loprencipe and A. Pantuso. A specified procedure for distress identification and assessment for urban road surfaces based on PCI. Coatings 7 (5), 2017, pp. 65.

[6] M. S. Snaith and D. M. Orr. Condition-based capital valuation of a road network. Proceedings of the Institution of Civil Engineers - Municipal Engineer 159 (2), 2006, pp. 91-95.

[7] G. Proctor and S. Varma. Incorporating asset valuation into transportation asset management financial plans. Federal Highway Administration, Washington DC, USA, 2016, Report FHWA-HIF-16-009.

[8] A. Pantuso, G. Loprencipe, G. Bonin et al. Analysis of pavement condition survey data for effective implementation of a network level pavement management program for kazakhstan. Sustainability 11 (3), 2019, pp. 901-916.

[9] L. Yao, Q. Dong, F. Ni et al. Effectiveness and cost-effectiveness evaluation of pavement treatments using life-cycle cost analysis. Journal of Transportation Engineering, Part B: Pavements 145 (2), 2019, pp. 04019006.

[10] New York State DoT. Network level condition assessment procedures. New York State Department of Transportation. New York, USA, 2010.

[11] Ministry of Transport PRC. JTG H20-2007: Highway performance assessment standards. China Communications Press. Beijing, China, 2007. 
[12] British Columbia Ministry of Transportation and Infrastructure. Pavement surface condition rating manual. Opus International Consultants (Canada) Limited. British Columbia, Canada, 2016.

[13] Highways Agency. HD 29/08: DMRB volume 7 pavement design and maintenance: Section 3 pavement maintenance assessment part 2 data for pavement assessment The Stationery Office. Norwich, UK, 2008.

[14] ASTM. D6433-09: Standard practice for roads and parking lots pavement condition index surveys. American Society for Testing and Materials (ASTM). Conshohocken, PA, USA, 2009.

[15] Austroads. Guide to pavement technology part 5: Pavement evaluation and treatment design Austroads Incorporated. Sydney, NSW, Australia, 2011.

[16] Austroads. Guide to asset management - technical information part 12: Pavements. Austroads Incorporated. Sydney, NSW, Australia, 2018.

[17] Ministry of Transport PRC. Interim measures for inspection and evaluation of highway maintenance quality. China Communication Press. Beijing, China, 1979.

[18] Ministry of Transport PRC. Standard for inspection and evaluation of highway maintenance quality. China Communication Press. Beijing, China, 1994.

[19] N. Li, K. Tom and K. Apple. Key pavement performance indicators and prediction models applied in a Canadian pms. In 8th International Conference on Managing Pavement Assets. (National Research Council (U.S.), Transportation Research Board, Universidad CatóLica De Chile and Ingenieria Dictus (eds)). Transportation Research Board of the National Academies, Santiago, Chile, 2011.

[20] W. Phang, M. Shahin and S. Kohn. Pavement condition rating and rehabilitation needs. Transportation Research Record 700, 1979, pp. 11-19.

[21] J. Hajek and W. Phang. Moving from subjective to objective evaluation of pavement performance. Ontario Ministry of Transportation, Ontario, Canada, 1986.

[22] N. Li, T. Kazmierowski, S. Tighe et al. Integrating dynamic performance prediction models into pavement management maintenance and rehabilitation programs. In 5th International Conference on Managing Pavements. (University of Washington and Washington (State) Department of Transportation (eds)). Engineering Professional Programs, University of Washington, Seattle Washington, United States, 2001.

[23] J. Hajek, T. Kazmierowsk and G. Musgrove. A switch to the international roughness index. Transportation Research Board 1643, 1998, pp. 116-124.
[24] Highway Research Board. The AASHO road test. National Academy of Sciences-National Research Council, Washington DC, USA, 1961, Report 1061.

[25] W. N. Carey Jr and P. E. Irick. The pavement serviceability-performance concept. Highway Research Board, Washington DC, USA, 1961, Report 250.

[26] United States Code. 23 U.S.C. 150: National goals and performance management measures, 2016.

[27] AASHTO. R43: Standard practice for quantifying roughness of pavements. AASHTO. Washington DC, USA, 2007.

[28] AASHTO. R48: Standard practice for determining maximum rut depth in asphalt pavements. AASHTO. Washington DC, USA, 2008

[29] AASHTO. R36: Standard practice for evaluating faulting of concrete pavements. AASHTO. Washington DC, USA, 2017.

[30] R. A. Cartwright and A. Pickett. TTS defects index preliminary analysis. Chris Britton Consultancy, Surrey, UK, 2004, Report Final version 2.

[31] S. Mcrobbie. Scanner condition indicator parameter thresholds and weightings. TRL Limited, Wokingham, UK, 2006, Report PPR 238.

[32] S. Mcrobbie, L. Walter, C. Read et al. Developing scanner road condition indicator parameter thresholds and weightings. TRL Limited, Wokingham, UK, 2007, Report PPR199.

[33] Department for Transport. Scanner surveys for local roads user guide and specification. TRL Limited, London, UK, 2011.

[34] Ministry of Transport PRC. JTG 5210-2018: Highway performance assessment standards. China Communications Press. Beijing, China, 2018.

[35] A. Simpson, G. Rada, B. Visintine et al. Development of next generation pavement performance measures. Federal Highway Administration, Washington DC, USA, 2013 Report FHWA-HIF-13-042.

[36] P. Marcelino, M. D. Lurdes Antunes and E. Fortunato. Comprehensive performance indicators for road pavement condition assessment. Structure and Infrastructure Engineering 14 (11), 2018, pp. 1433-1445.

[37] Y. Dong, Y. Shao, X. Li et al. Forecasting pavement performance with a feature fusion lstm-bpnn model. In Proceedings of the 28th ACM International Conference on Information and Knowledge Management. (Jiang M and Qiao M (eds)). Association for Computing Machinery, Beijing, China, 2019, pp. 1953-1962. 